\title{
Possible Complications of Endodontic Treatments
}

\author{
Gabriele Miccoli ${ }^{1}$, Marco Seracchiani ${ }^{2}$, Alessio Zanza ${ }^{3}$, Andrea Del Giudice ${ }^{4}$, Luca Testarelli ${ }^{5}$
}

The Journal of Contemporary Dental Practice (2020): 10.5005/jp-journals-10024-2811

Endodontic treatments tend to have very high successful rates in spite of the fact that they are quite an invasive, difficult procedure, especially in complex anatomies. Some complications can arise during or after a root canal procedure due to poor understanding of the anatomy and iatrogenic errors mainly during instrumentation. While some of these problems can be anticipated, many can never really be predicted. ${ }^{1-3}$ As a consequence, retreatment has become a well-defined field of knowledge and expertise in endodontics, since most complications can be solved with proper surgical or nonsurgical retreatments. ${ }^{4}$ Reinfection or persistent infections: Root canal treatment can fail due to a persistent bacterial infections or reinfections. These include inadequate shaping, cleaning, and obturation procedures, poor restorations, and exposure through a fracture. The remedies are dependent on the primary cause of the reinfection and the affected parts. Persistent infection is the major cause of short-term endodontic failures. ${ }^{5}$

Missed root canals: Canal anatomy can be complex and variable. Clinicians can sometimes miss a canal in a complex tooth structure. The increasing use of more advanced 3D radiographic images (CBCT) is very helpful to reduce these errors. ${ }^{6}$ Fractured root or crown: The carious lesions and the endodontic procedure (access cavity and canal enlargement) leaves make it brittle and fragile. Fractures on the crown or root can be detected before, during, or after the root canal. Crown fracture is the major cause of long-term endodontic failures. ${ }^{7}$ Depending on the extent and severity of the fracture, the dentist will advise on whether extraction (and placement of an implant) or a filling is the correct option. Fractured instruments: This happens especially if the canal is complex and curved, and instrumentation stresses become greater than the mechanical resistance of the instruments. Being a quite frequent complication in common practice, many studies have been published to understand the mechanisms of these iatrogenic errors and instruments' resistance (cyclic fatigue and torsional tests). A fractured instrument is a potential factor for failure when it negatively affects the correct shaping and cleaning procedure. Clinicians should be very careful in avoiding overstressing, mainly the rotating nickel-titanium instruments of greater tapers..$^{8,9}$

Postoperative pain and discomfort: These are common symptoms immediately after a root canal procedure, but should resolve within a few weeks. ${ }^{10}$ If a tooth stays or becomes symptomatic after months/years, this is a sign of treatment failure. Symptoms related to discomfort or pain on chewing, aching, and so forth are generally an indication of periradicular inflammation or infection. It must be remembered that bony healing takes time and that a tooth that feels "different" on biting may be en route to healing; this should be confirmed clinically and radiographically. Pain on release may indicate a cracked tooth. A bad taste may indicate a draining abscess. Occasionally, a patient reports sensitivity to cold or heat; this is most likely related to an adjacent
1-5 Department of Oral and Maxillo-Facial Sciences, Sapienza University
of Rome, Roma, Italy
Corresponding Author: Luca Testarelli, Department of Oral and
Maxillo-Facial Sciences, Sapienza University of Rome, Rome, Italy,
Phone: +39 3381504134, e-mail: luca.testarelli@uniroma1.it
How to cite this article: Miccoli G, Seracchiani M, Zanza A, et al.
Possible Complications of Endodontic Treatments. J Contemp Dent
Pract 2020;21(5):473-474.
Source of support: Nil
Conflict of interest: None

untreated tooth but could be an indication of a missed vital canal in a treated tooth. ${ }^{1}$

Post-treatment: As with all dental procedures, complications may occur after root canal treatment. However, the incidence of long-term postoperative complications appears to be lower than for the alternatives, single tooth implants, and fixed dental prostheses. $^{11}$

In conclusion, the patient history, clinical findings, and radiographic examinations are fundamental metrics of root canal treatment outcomes assessment and data they provide should never be underestimated.

\section{References}

1. Bergenholtz G. Assessment of treatment failure in endodontic therapy. J Oral Rehabil 2016;43(10):753-758. DOI: 10.1111/joor.12423.

2. Plotino G, Grande NM, Mazza C, et al. Influence of size and taper of artificial canals on the trajectory of NiTi rotary instruments in cyclic fatigue studies. Oral Surg Oral Med Oral Pathol Oral Radiol Endod 2010;109(1):e60-e66. DOI: 10.1016/j.tripleo.2009.08.009.

3. Gambarini G, Seracchiani M, Piasecki L, et al. Measurement of torque generated during intracanal instrumentation in vivo. Int Endod J 2019;52(5):737-745. DOI: 10.1111/iej.13042.

4. Alghamdi F, Alhaddad AJ, Abuzinadah S. Healing of periapical lesions after surgical endodontic retreatment: a systematic review. Cureus 2020;12(2):e6916. DOI: 10.7759/cureus.6916.

5. Prada I, Mico-Munoz P, Giner-Lluesma T, et al. Influence of microbiology on endodontic failure. Literature review. Med Oral Patol Oral Cir Bucal 2019;24(3):e364-e372. DOI: 10.4317/medoral.22907.

6. Gambarini G, Piasecki L, Miccoli G, et al. Cone-beam computed tomography in the assessment of periapical lesions in endodontically treated teeth. Eur J Dent 2018;12(1):136-143. DOI: 10.4103/ejd. ejd_320_17.

7. Figueiredo FE, Martins-Filho PR, Faria-E-Silva AL. Do metal postretained restorations result in more root fractures than fiber post-retained restorations? A systematic review and meta-analysis. J Endod 2015;41(3):309-316. DOI: 10.1016/j.joen.2014.10.006.

8. Gambarini G, Piasecki L, Miccoli G, et al. Classification and cyclic fatigue evaluation of new kinematics for endodontic instruments. Aust Endod J 2019;45(2):154-162. DOI: 10.1111/aej.12294. 
9. Gambarini G, Miccoli G, Seracchiani M, et al. Fatigue resistance of new and used nickel-titanium rotary instruments: a comparative study. Clin Ter 2018;169(3):e96-e101. DOI: 10.7417/T.2018. 2061.

10. Gambarini G, Di Nardo D, Miccoli G, et al. The influence of a new clinical motion for endodontic instruments on the incidence of postoperative pain. Clin Ter 2017;168(1):e23-e27. DOI: 10.7417/ CT.2017.1977.

11. Guarnieri R, Di Nardo D, Gaimari G, et al. Short vs. standard laser-microgrooved implants supporting single and splinted crowns: a prospective study with 3 years follow-up. J Prosthodont 2019;28(2):771-779. DOI: 10.1111/jopr.12959. 